\title{
Electronic and magnetic properties of bulk Cr tips for scanning tunneling spectroscopy
}

\author{
F. Donati, ${ }^{1,2}$ G. Fratesi, ${ }^{3}$ L. Ning, ${ }^{4}$ A. Brambilla,${ }^{5}$ M. I. Trioni, ${ }^{6}$ A. Li Bassi, ${ }^{1,7}$ C. S. Casari, ${ }^{1,7}$ and M. Passoni ${ }^{1}$ \\ ${ }^{1}$ Dipartimento di Energia, CNISM and NEMAS - Center for NanoEngineered Materials and Surfaces, Politecnico di Milano, \\ via Ponzio 34/3, I-20133 Milano, Italy \\ ${ }^{2}$ Institute of Condensed Matter Physics (ICMP), Ecole Polytechinque Fédérale de Lausanne (EPFL), Station 3, CH-1015, Switzerland \\ ${ }^{3}$ ETSF, CNISM, Dipartimento di Scienza dei Materiali, Università di Milano-Bicocca, Via Cozzi 53, I-20125 Milano, Italy \\ ${ }^{4}$ Department of Physics, Anhui Normal University, Wuhu, Anhui 241000, China \\ ${ }^{5}$ Dipartimento di Fisica, CNISM and NEMAS - Center for NanoEngineered Materials and Surfaces, Politecnico di Milano, \\ piazza Leonardo da Vinci 32, I-20133 Milano, Italy \\ ${ }^{6}$ CNR - National Research Council of Italy, ISTM, via Golgi 19, I-20133 Milano, Italy \\ ${ }^{7}$ Center for Nano Science and Technology @ Polimi, Istituto Italiano di Tecnologia, Via Pascoli 70/3, I-20133 Milano, Italy
}

(Received 2 November 2012; revised manuscript received 3 April 2013; published 26 June 2013)

\begin{abstract}
The electronic and magnetic properties of bulk Cr tips for scanning tunneling microscopy have been investigated by means of density functional theory (DFT) calculations and scanning tunneling spectroscopy (STS) measurements. Spin-resolved densities of states (DOS) were calculated for model tips, i.e., Cr adatoms and clusters on ideal $\mathrm{Cr}$ surfaces. STS measurements on $\mathrm{Au}(111)$ and $\mathrm{Si}(111)-7 \times 7$ have been interpreted by modeling the tunneling process in a Wentzel-Kramers-Brillouin approximation in order to ascertain the role of tip DOS features. Calculated spin-resolved electronic properties of $\mathrm{Cr}$ tips have been used to revisit spin-polarized (SP) STS measurements from the literature. The agreement between experimental findings and DFT calculations confirms the relevance of the knowledge of tip electronic properties and of an appropriate model for the tunneling process in the interpretation of STS and SP-STS data.
\end{abstract}

DOI: 10.1103/PhysRevB.87.235431

PACS number(s): 07.79.Cz, 68.37.Ef, 75.50.Ee, 72.25.Ba

\section{INTRODUCTION}

Since their invention, scanning tunneling microscopy (STM) and spectroscopy (STS) have been performed using tunneling tips fabricated with a great variety of materials. So far, the most commonly adopted tips are prepared by electrochemical etching of $\mathrm{W}$ wires. ${ }^{1}$ However, for some specific applications, such as spin-polarized scanning tunneling microscopy (SP-STM) and spectroscopy (SP-STS), other materials are required. Ferromagnetic or antiferromagnetic metals have been exploited to obtain probes with spin sensitivity. Ferromagnetic metals typically provide a higher spin sensitivity, but antiferromagnetic tips are usually preferred because they do not exhibit significant perturbing stray field. In particular, $\mathrm{Cr}$ is the only metal with a (bulk) Néel temperature (311 K) above room temperature, making it interesting for SP-STM experiments in a wide temperature range. In the literature, the most common procedure to produce SP-STM tips is to coat $\mathrm{W}$ tips with a thin film of magnetic material such as $\mathrm{Fe}$, $\mathrm{Gd}$, or $\mathrm{Cr}^{2-4}$ This method allows the experimenter to have good control of material purity and tip properties; however, it also requires specific in situ facilities for tip cleaning and magnetic film evaporation, which might not be available in all experimental setups. Alternatively, antiferromagnetic bulk tips can be more easily prepared by ex situ electrochemical etching ${ }^{5}$ and therefore represent an interesting alternative to coated W tips.

Proposed since the invention of the SP-STM technique, ${ }^{6}$ bulk $\mathrm{Cr}$ tips have been demonstrated to provide spin contrast in SP-STM ${ }^{7}$ and SP-STS experiments ${ }^{8,9}$ only in recent years. Such works pointed out the sensitivity of these probes, but the magnetic behavior of bulk $\mathrm{Cr}$ tips, i.e., the sign and the energy dependence of spin polarization, as well as the tip magnetic easy axis and response to magnetic field, seems to differ among the various experiments. Although bulk Cr tips properties have been explored for a single structural configuration by $a b$ initio calculations, ${ }^{10}$ a detailed investigation supported by experimental results is still missing.

In particular, quantities such as the tip density of states (DOS) and spin polarization $\left(P_{t}\right)$ are crucial to correctly extract the sample properties from STS and SP-STS experiments. Alternative methods face different problems in this context. On the one hand, tip apex properties can be obtained from STS experiments only on well-known surfaces, ${ }^{11,12}$ but still the interaction between tip and sample can modify the apex structure and composition. On the other hand, $a b$ initio calculations provide access to a great number of tip properties, but they rely on a correct input structure, which is also in principle unknown and can vary during experiments. The intrinsic limits of the two approaches suggest to adopt a combined strategy, namely simulating the tip structure and electronic properties and comparing the results with the tip DOS identified from STS and SP-STS experiments on wellknown surfaces.

Here, we report on the investigation of bulk $\mathrm{Cr}$ tip properties by a combination of ab initio DFT calculations and STS measurements (as detailed in Sec. II). By using different structural configurations, we calculated the DOS of bulk $\mathrm{Cr}$ tips at the tip apex, which is known to be the relevant quantity for STM and STS experiments (reported in Sec. III A). STS experiments have been performed on $\mathrm{Si}(111)-7 \times 7$ and $\mathrm{Au}(111)$ surfaces to identify the relevant tip features. In order to match the pieces of information between DFT calculations and STS measures, we simulated STS experiments by means of a Wentzel-Kramers-Brillouin (WKB) model using the calculated tip DOS as the input (see Sec. III B). Eventually, DFT calculations were used to interpret Cr tip SP-STS data from the literature as discussed in Sec. III C. Our study reveals 
how the knowledge of the tip DOS combined with the use of a proper tunneling model can be effective for a quantitative interpretation of STS experiments.

\section{THEORETICAL AND EXPERIMENTAL DETAILS}

Numerical simulations based on DFT were performed to obtain the DOS of $\mathrm{Cr}$ tips modeled here as $\mathrm{Cr}$ adatoms on hollow sites of $\operatorname{Cr}(100)$, (110), and (111) surfaces. In the calculations we used the PBE exchange-correlation functional ${ }^{13}$ and treated the surface as an antiferromagnetic metal slab in a periodically repeated supercell. We neglect the spin density wave which modulates the layer antiferromagnetism in bulk $\mathrm{Cr}^{14}$ and restrict ourselves to collinear magnetic configurations of $\mathrm{Cr}$ tips, recognizing that possible noncollinear configurations might emerge in systems of reduced dimension owing to frustration of antiferromagnetic interactions. Such a limitation however does not prevent a qualitative analysis like the one made possible by the presented methodology. A plane wave basis set and the projector augmented wave method $^{15,16}$ as implemented in the VASP simulation package ${ }^{17}$ were used with a cutoff of $227 \mathrm{eV}$. This results in a $\mathrm{Cr}$ bulk lattice parameter of $2.85 \AA$, in excellent agreement with experimental findings and previous calculations, and atomic magnetic moment of $\pm 1.06 \mu_{B}$, overestimated as expected from PBE calculations. ${ }^{18-20}$ We adopted $(3 \times 3), c(4 \times 3)$, and $(3 \times 3)$ surface supercells with 6,5 , and 15 bulk layers to treat (100), (110), and (111) surfaces, respectively, so that all slabs have a similar thickness of about $10 \AA$. In all cases, a $3 \times 3 k$-point grid was used. The vacuum separation between adjacent slabs was at least $10 \AA$. The topmost 3 layers were allowed to relax together with the adatom with a threshold on forces of $0.01 \mathrm{eV} / \AA$. The DOS was computed on a denser $5 \times 5 k$-point mesh and evaluated on the apex atom of the structure.

An Omicron VT-SPM apparatus was used for preparing and measuring the surfaces. All the measurements were performed at room temperature. The $\mathrm{Si}(111)-7 \times 7$ surface was prepared by several flash annealing cycles at $1500 \mathrm{~K}$ by direct current heating. Large terraces with a regular, contamination-free $7 \times 7$ reconstruction were obtained. The $\mathrm{Au}(111)$ surface (a commercial substrate of evaporated gold on mica) was

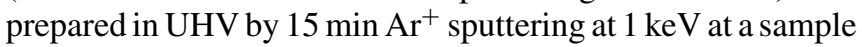
temperature of $800 \mathrm{~K}$, which was kept for at least $10 \mathrm{~min}$ after the sputtering. After several cycles, a clean reconstructed surface was obtained with a low level of contaminations. ${ }^{21}$ Bulk Cr tips were prepared through electrochemical etching as described in Ref. 7. To remove residual oxide layers from the tip apex, voltage pulses were applied while scanning on $\mathrm{Au}(111)$ until a stable behavior was observed, but avoiding tip indentation into the surface. Differential conductance $d I / d V$ spectra were acquired by means of a lock-in amplifier, applying a modulation $V_{\text {mod }}=50 \mathrm{mV}$ peak-to-peak after the feedback loop was opened at a given voltage-current $(V, I)$ set point.

\section{RESULTS AND DISCUSSION}

\section{A. Simulations of $\mathrm{Cr}$ tip models}

Following a commonly adopted approach, ${ }^{10,22,23}$ simple model structures are assumed to model the real STM tip apex.

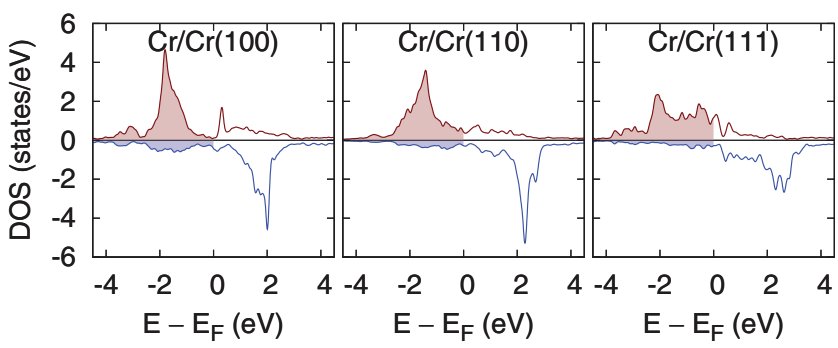

FIG. 1. (Color online) Spin-resolved DOS of bulk Cr tips for the three structural configurations considered. The minority DOS is reversed and the Fermi level $E_{\mathrm{F}}$ was set as energy reference.

This allows one to extract the main features of tip DOS and spin polarization from a number of possible ideal structures to be compared with measurements performed with real tips, whose structures are unknown and can change during the experiments. Since a minimal protrusion is needed to perform STM with sufficient spatial resolution, we do not take into account the model structures represented by the bare $\mathrm{Cr}(100)$, (110), and (111) surfaces. As the simplest tip structures, we simulate here the case of a $\mathrm{Cr}$ adatom on $\mathrm{Cr}(100)$, (110), and (111). Notice that only one spin-collinear magnetic configuration with nearest-neighbor antiferromagnetism needs to be considered in all three cases. The adatom DOS are reported in Fig. 1. In all cases, the spin polarization of the adatom is evident, with an almost filled majority-spin band $(\uparrow)$ and an almost empty minority-spin one $(\downarrow)$. This results in a large adatom magnetic moment, 2.53, 3.16, and $3.32 \mu_{B}$ for the three cases, respectively. While the actual figures are presumably overestimated by DFT-PBE, the strong enhancement of the atomic magnetization we compute is a well established feature of the Cr surface. ${ }^{19,20,24}$

To better discuss the observed experimental features it is interesting to look at the total DOS of the three tip models, which is reported in Fig. 2(a). The $\mathrm{Cr} / \mathrm{Cr}(100)$ DOS is dominated by a sharp peak at about $+0.3 \mathrm{eV}$ and also
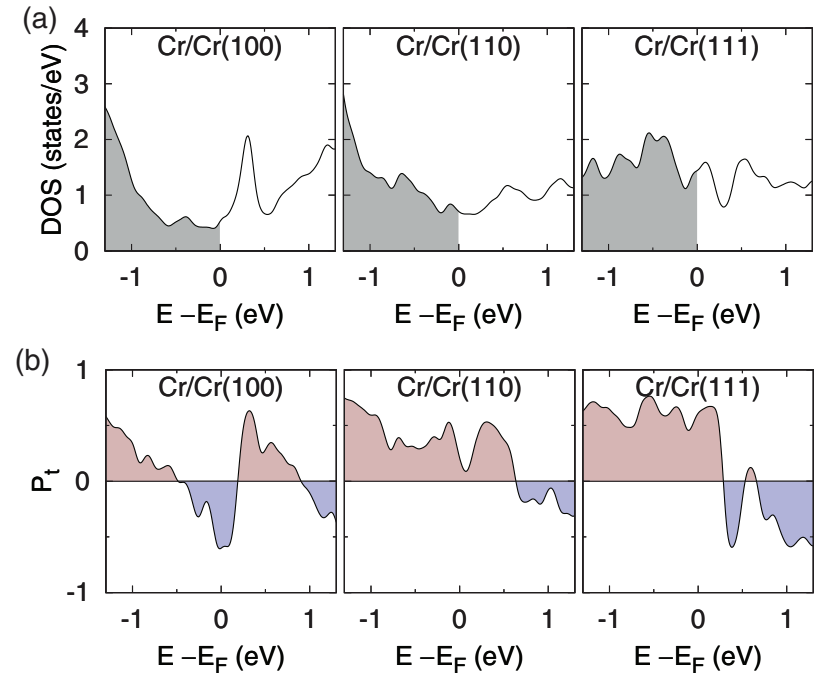

FIG. 2. (Color online) (a) Spin-summed DOS of bulk Cr tips for the three structural configurations considered. (b) Spin polarization $P_{t}$, see Eq. (1), as a function of energy. 
displays a smaller feature at $-0.4 \mathrm{eV}$. Similar results were also obtained by Ferriani et al. ${ }^{10}$ Conversely, $\mathrm{Cr} / \mathrm{Cr}(110)$ and $\mathrm{Cr} / \mathrm{Cr}(111)$ DOS show three broad peaks, which are found at the energies of $+0.6 \mathrm{eV},-0.1 \mathrm{eV}$, and $-0.7 \mathrm{eV}$ for $\mathrm{Cr} / \mathrm{Cr}(110)$ and at $+0.5 \mathrm{eV},+0.1 \mathrm{eV}$, and $-0.5 \mathrm{eV}$ for $\mathrm{Cr} / \mathrm{Cr}(111)$. All these peculiar features, being close to the Fermi level, should produce relevant effects in the STS spectra, as will be discussed in Secs. III B and III C.

To detect a magnetic signal in SP-STM experiments, a large asymmetry in the two spin channels close to the Fermi level is required. ${ }^{25}$ It is then useful to look at the spin polarization of the tip, $P_{t}$, which is defined as

$$
P_{t}=\frac{\mathrm{DOS}_{\uparrow}-\mathrm{DOS}_{\downarrow}}{\mathrm{DOS}_{\uparrow}+\mathrm{DOS}_{\downarrow}},
$$

and is reported in Fig. 2(b). $\mathrm{Cr} / \mathrm{Cr}(100)$ and $\mathrm{Cr} / \mathrm{Cr}(111)$ have large and opposite spin polarizations at the Fermi level, $P_{t}=-0.6$ and +0.6 , respectively [a smaller value was found for $\mathrm{Cr} / \mathrm{Cr}(110)$, for which $\left.P_{t}=+0.2\right]$. These observations indicate that both positive and negative large values of $P_{t}$ are possible depending on the tip structure, in agreement with recent experimental findings. ${ }^{\text {? }}$

\section{B. Scanning tunneling spectroscopy with bulk $\mathrm{Cr}$ tips}

In order to identify $\mathrm{Cr}$ tip features from STS data and to compare them to DFT calculations, we follow the formal apparatus used in Refs. 11 and 26 to model the tunneling current in the WKB framework:

$$
I(V) \propto \int_{0}^{e V} \rho_{s}(E) \rho_{t}(E-e V) T(E, V) d E,
$$

where $I$ is the tunneling current, $V$ is the applied voltage, $E$ is the energy of the sample state (where we take the zero at the Fermi level), $\rho_{s, t}$ are the sample/tip density of states at their respective surface (outermost atom), and $T(E, V)$ is the transmission coefficient of the tunneling junction. This approach allows one to capture the main aspects of tunneling and to identify sample and tip electronic features without performing more demanding full transport calculations. ${ }^{11,26,27}$ Assuming a $1 \mathrm{D}$ trapezoidal potential barrier along the direction perpendicular to the surface, the transmission coefficient can be expressed as

$$
T(E, V)=\exp \left[-2 z \sqrt{\frac{2 m}{\hbar^{2}}[\phi+e V / 2-f(E)]}\right] .
$$

Here, $m$ is the free electron mass, $z$ is the tip-sample distance, $\phi$ is the average between tip and sample work functions, and $f(E)$ is a function which depends on surface properties. For example, in systems where tunneling is strongly dominated by the contribution of states with zero parallel wave vector $(\bar{\Gamma})$, it is common to assume ${ }^{26} f(E)=E$, while $f(E)=E_{0}$ is more appropriate for Shockley states $\left(E_{0}\right.$ is the energy value of the surface state at $\bar{\Gamma}) .{ }^{27-29}$ Although such formulation of the tunneling coefficient cannot account for 3D aspects such as tip apex symmetry and the band structure of tip and sample ${ }^{29}$ this simple description allows one to carry out a direct identification of tip and sample electronic contributions.
The current derivative with respect to $V$,

$$
\begin{aligned}
& \frac{d I}{d V} \propto \rho_{s}(e V) \rho_{t}(0) T(e V, V) \\
& \quad+\int_{0}^{e V} \rho_{s}(E) \frac{d}{d V}\left[\rho_{t}(E-e V) T(E, V)\right] d E,
\end{aligned}
$$

represents the quantity typically measured in STS experiment. The energy dependence of $d I / d V$ on tip DOS is contained in the second term of Eq. (4). In the case of constant tip DOS this term produces a smooth background contribution and is often neglected. If contrarily the tip DOS is nonconstant, this term could give rise to extra features in $d I / d V$ spectra. ${ }^{11}$ These are of particular interest in our work because they can be used for identifying tip properties. In particular, when the sample and tip DOS are known from ab initio calculations, it is possible to quantitatively simulate STS spectra by making use of Eqs. (2) or (4) and to compare the results to experimental $d I / d V$ curves. An approximate approach can instead be adopted if a detailed knowledge of sample or tip DOS is missing. In the limit of low biases, Eq. (4) reduces to 26

$$
\frac{d I}{d V} \approx \rho_{s}(e V) \rho_{t}(0) T(e V, V)+\rho_{s}(0) \rho_{t}(-e V) T(0, V) .
$$

In this form, one recognizes a direct contribution of tip DOS with a reversed energy scale. In other words, tip DOS features at an energy $e V$ could be found in $d I / d V$ at the voltage $-V$ overlapped to the sample DOS features. A basic knowledge of the sample DOS can thus allow one to identify the presence of tip features in STS spectra. As this form is strictly valid for low biases, its extension to a wide energy range has to be considered as an approximation. Summarizing, Eq. (5) represents a valid tool for qualitatively interpreting STS spectra in the presence of a nonconstant tip DOS when a detailed knowledge of the sample is not available. ${ }^{30}$

We first apply the approximate approach of Eq. (5) to the $\operatorname{Si}(111)-7 \times 7$ surface, which presents a peculiar reconstruction with seven nonequivalent sites. ${ }^{31}$ Because of the relatively large separation between the surface atoms (about $1 \mathrm{~nm}$ ), this sample allows one to test STS capability with atomic resolution. ${ }^{32}$ As described in the Takanayagi model, ${ }^{33}$ the 12 topmost atoms, called adatoms, occupy four nonequivalent sites, namely corner unfaulted $(\mathrm{CoU})$, center unfaulted $(\mathrm{Ce} U)$, corner faulted $(\mathrm{CoF})$, and center faulted $(\mathrm{CeF})$. Six other atoms, called rest atoms, lie below the adatom plane and occupy two nonequivalent sites, namely rest unfaulted $(R U)$ and faulted $(R F)$ and are more difficult to be resolved by STM. ${ }^{34}$ Vacancies of surface atoms referred to as corner holes $(\mathrm{CoH})$ represent the seventh nonequivalent site. In Fig. 3(a), we show an STM image taken with a bulk Cr tip. As visible from the line profile in Fig. 3(b), bulk Cr tips reveal a peculiar sensitivity in imaging rest atoms, resolving them as isolated protrusions. ${ }^{7}$ Since this resolution is hardly obtained with $\mathrm{W}$ tips,${ }^{34}$ it was thought that it could be a direct consequence of the Cr electronic structure. To address this point, in Figs. 3(c)-3(f) we show STS spectra acquired with $\mathrm{Cr}$ and $\mathrm{W}$ tips on four different nonequivalent sites of the $7 \times 7$ reconstructed surface. To remove differences in $d I / d V$ amplitude introduced by the different set point and the characteristic transmission coefficient exponential behavior [see Eqs. (2)-(4)], we apply the normalization method described in Ref. 26. For all the 

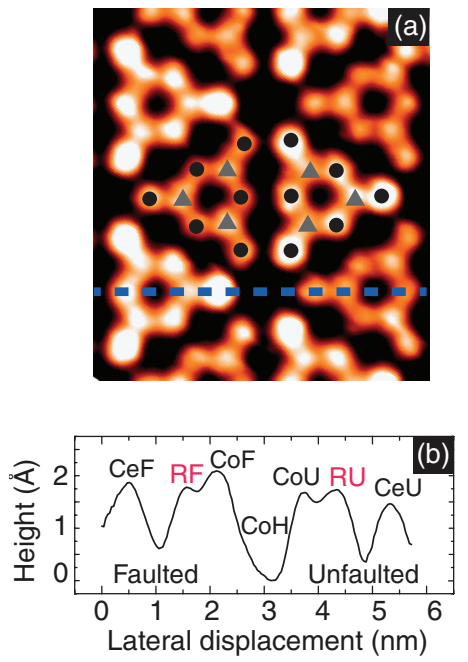
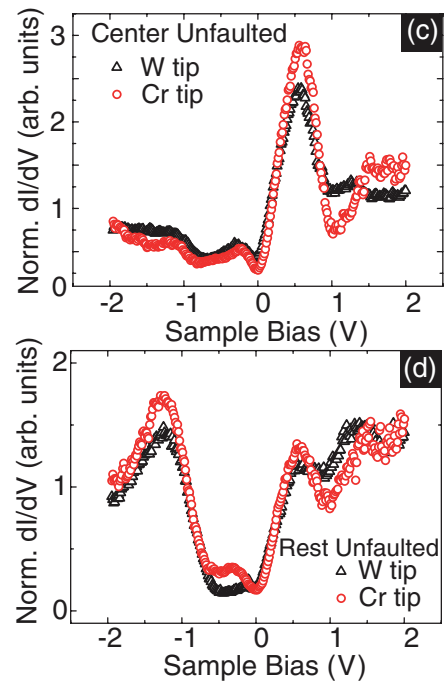
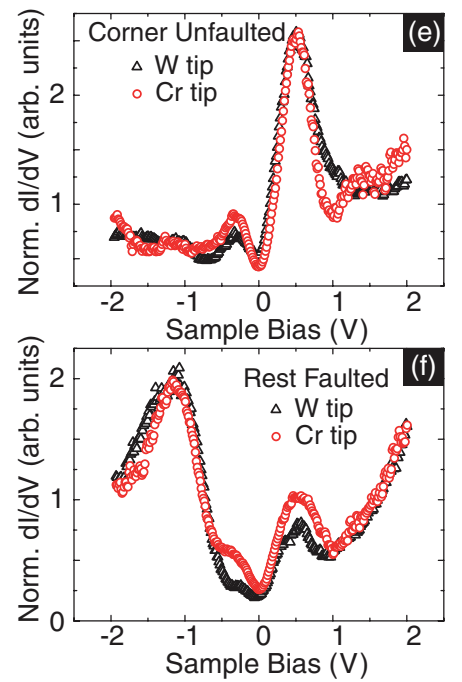

FIG. 3. (Color online) (a) STM image of Si(111) surface obtained using a bulk Cr tip. (Set point: $V=-1.5 \mathrm{~V}, I=2.2 \mathrm{nA}$.) Black dots and gray triangles represent adatoms and rest atom positions, respectively. The image distortion due to thermal drift has been corrected to match the expected surface symmetry. (b) Profile of $\mathrm{Si}(111)-7 \times 7$ unit cell along the dashed blue line in (a). (c)-(f) $d I / d V$ curves obtained with bulk Cr tip (red circles; set point: $V=1 \mathrm{~V}, I=1 \mathrm{nA}$ ) and W tip (black triangles; set point: $V=1 \mathrm{~V}, I=0.5 \mathrm{nA}$ ).

measured sites, peaks at about $+0.5 \mathrm{~V}$ and $-0.3 \mathrm{~V}$ are visible while another feature at $-1.2 \mathrm{~V}$ is visible for $R F$ and $R U$ sites in agreement with the literature. ${ }^{32,35-37}$ Some differences are visible between the $\mathrm{Cr}$ and $\mathrm{W}$ tip spectra. A clear enhancement of the signal at $-0.3 \mathrm{~V}$ is visible in $\mathrm{Cr}$ spectra of both $R U$ and $R F$, while only for $R F$ a further small enhancement is detected at $+0.5 \mathrm{~V}$. Other differences were observed in $\mathrm{CeU}$ and $\mathrm{CoU}$ spectra, where $\mathrm{Cr}$ tips produce an enhancement of the signal at the biases of $+0.5 \mathrm{~V}$ and $-0.3 \mathrm{~V}$, respectively. Interpreting these results in the spirit of Eq. (5), we conclude that the differences between $\mathrm{W}$ and $\mathrm{Cr}$ tip spectra can be attributed to the differences in the tip DOS. Thus, the enhancement in Cr tip STS spectra at the bias of $+0.5 \mathrm{~V}$ and $-0.3 \mathrm{~V}$ is the signature of two tip features at $E=-0.5 \mathrm{eV}$ and $+0.3 \mathrm{eV}$, respectively, not observed for the $\mathrm{W}$ tip. Recalling now the DOS of our model tips as computed by DFT and reported in Fig. 2(a), we notice that a similar structure is indeed found in the case of $\mathrm{Cr} / \mathrm{Cr}(100)$. We conclude that such a configuration might be the closest to the real $\mathrm{Cr}$ tip apex structure of the experiment from an electronic point of view.

The STS signal enhancement measured at $-0.3 \mathrm{~V}$ on $R F$ and $R U$ is of particular interest. As reported in Ref. 7, Cr bulk tips showed a peculiar sensitivity in imaging rest atoms when a negative bias of $-1.5 \mathrm{~V}$ was used. A similar effect was observed for InAs tips, and ascribed to the bulk electronic properties of the tip. ${ }^{38}$ In that case, it was found that the conduction surface-projected gap acts as an energy filter, which suppresses the adatoms' LDOS contribution to the tunneling current while leaving the rest atoms' signal, thus allowing the rest atoms to be imaged as isolated protrusions. As well as for InAs tips, the peculiar sensitivity of $\mathrm{Cr}$ tips could be due to its electronic structure. In particular, since the $\mathrm{Cr}$ tip DOS generates an extra feature at $-0.3 \mathrm{~V}$ in $d I / d V$, integrating such contribution in Eq. (2) would provide an increase in the tunneling current. Consequently, the feedback loop would induce the tip to further retract from the surface, thus imaging the rest atoms as isolated protrusion.

As a last remark for this analysis of the $\operatorname{Si}(111)-7 \times 7$ surface, some variations in the signal amplitude due to the tip features are visible depending on the atomic site. We speculate that this effect could be due to specific 3D aspects of the tunneling process, such as the spatial extent of the tip apex and the matrix element dependence on the tip and the surface orbital symmetry. ${ }^{29,30}$ In particular, nonequivalent atomic sites within the $\operatorname{Si}(111)-7 \times 7$ reconstruction differ not only by their DOS but also by the energy-dependent spatial decay of their electronic states, which can introduce a further modulation in the current intensity.

We then analyze STS spectra on $\mathrm{Au}(111)$ by the more precise Eq. (4) which allows us to identify $\mathrm{Cr}$ tip DOS features in a more quantitative way. The $\mathrm{Au}(111)$ surface is characterized by a nearly-free-electron Shockley state having a parabolic dispersion with electron momentum. Integrating over the surface Brillouin zone, this state generates a step in the surface DOS with the onset at $-0.5 \mathrm{eV}$. This onset is typically observed in STS spectra as a step in differential conductance, superposed to a smooth decreasing background due to the energy dependence of the transmission coefficient. ${ }^{39}$ Given its simple DOS structure, such a surface is particularly suitable to identify tip features in a quantitative way.

In Fig. 4(a) we show a $d I / d V$ curve acquired with a bulk $\mathrm{Cr}$ tip on $\mathrm{Au}(111)$. In addition to the well-known onset at -0.5 $\mathrm{eV}$, a decreasing background is observed for $V<0$. Such a trend continues up to about $1 \mathrm{~V}$, where the signal begins to increase again, and is characterized by a change of slope at $V=-0.05 \mathrm{~V}$. In order to ascertain the origin of the observed features, the tunneling current is simulated through Eq. (2) by employing several model tip DOS. As the sample DOS, we use a Heaviside function with the onset at $-0.5 \mathrm{eV}$ and a broadening of $0.15 \mathrm{eV}$ to take into account finite lifetime of 


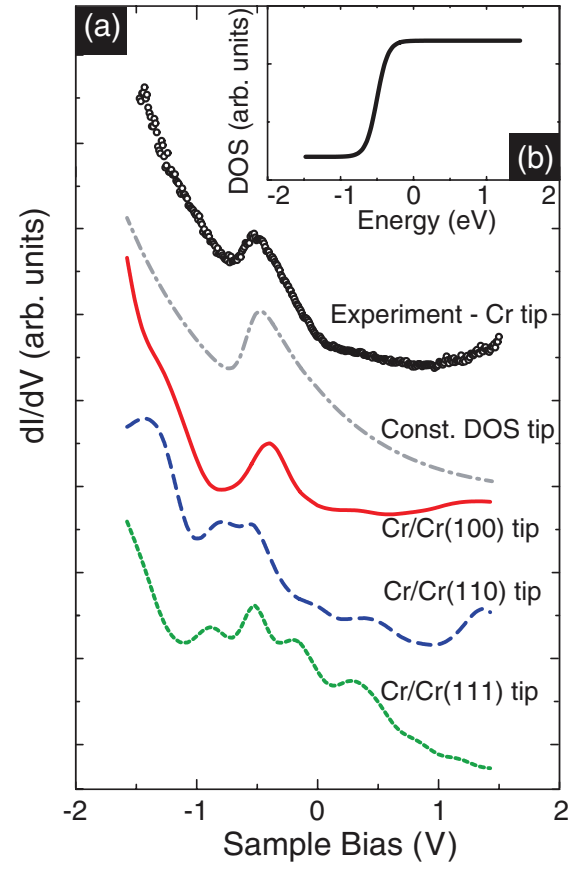

FIG. 4. (Color online) (a) Experimental (black dotted line; setpoint: $V=1 \mathrm{~V}, I=1 \mathrm{nA}$ ) versus calculated $d I / d V$ curves obtained numerically differentiating $I$ in Eq. (2) assuming either a constant tip DOS (gray dash-dotted line) or one as computed for $\mathrm{Cr} / \mathrm{Cr}(100)$ (red solid line), $\mathrm{Cr} / \mathrm{Cr}(110)$ (blue dashed line), and $\mathrm{Cr} / \mathrm{Cr}(111)$ (green dotted line); see Sec. III A. (b) Model Au(111) DOS used in Eq. (2) for simulating the $d I / d V$ curves shown in (a).

the electron states ${ }^{40}$ and lock-in modulation; see Fig. 4(b). As explained above, we model the transmission coefficient using the expression in Eq. (3) with $\phi=5 \mathrm{eV}$ as the tip-sample effective work function and $E_{0}=-0.5 \mathrm{eV}$ as the onset of the Shockley state. $d I / d V$ is then obtained by numerically differentiating $I$ in Eq. (2). First we focus on the simulated $d I / d V$ for a tip with constant DOS, reported in Fig. 4(a). The conductance step and the decreasing background are correctly reproduced but the change of slope at $-0.05 \mathrm{~V}$ and the intensity increase at $V>1 \mathrm{~V}$ are not (see Fig. 4). This points out the need of taking into account the energy dependence of the tip DOS. We then calculate $d I / d V$ curves by using the DOS of $\mathrm{Cr} / \mathrm{Cr}(100), \mathrm{Cr} / \mathrm{Cr}(110)$, and $\mathrm{Cr} / \mathrm{Cr}(111)$. Evidently, the case of $\mathrm{Cr}(100)$ best reproduces not only the conductance step and the smooth decreasing background but also the change of slope at $-0.05 \mathrm{~V}$ and the intensity increase at $V>1 \mathrm{~V}$. No signature of the peak at $+0.3 \mathrm{eV}$ in the $\mathrm{Cr} / \mathrm{Cr}(100)$ DOS can be resolved in the $d I / d V$ spectra. As described in Eq. (5), features in the tip DOS are expected to be visible in $d I / d V$ spectra at a reversed energy scale. Since the peak at $+0.3 \mathrm{eV}$ in tip DOS would produce a feature at $-0.3 \mathrm{~V}$ in the $d I / d V$ spectra, the overlap with the broad conductance step in the sample DOS at $-0.5 \mathrm{eV}$ prevents its direct detection. We note that these features were observed using different tips, in contrast to a number of other features that were measured with less stable tip configurations and that could not be reproduced.

As already pointed out in Ref. 10, the tip electronic properties of a particular metal are similar when similar symmetries are involved. Having identified $\mathrm{Cr} / \mathrm{Cr}(100)$ as a

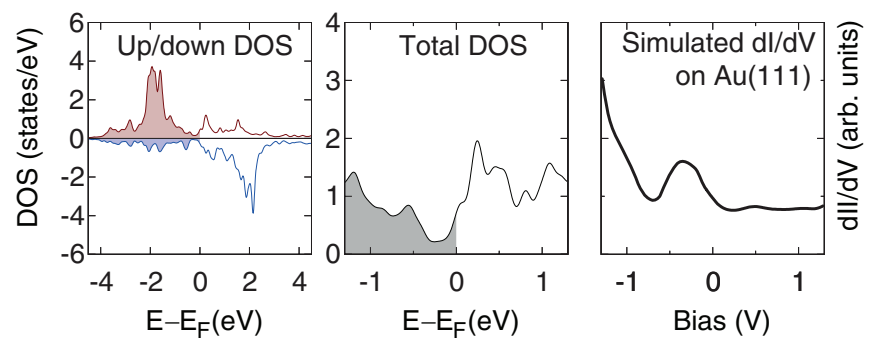

FIG. 5. (Color online) Bulk Cr tip modeled as a 5-atom pyramid on $\mathrm{Cr}(100)$. From left to right: Spin-resolved DOS, total DOS, simulated $d I / d V$ for the model $\mathrm{Au}(111)$ surface.

good candidate to model the real Cr apex in STS experiments, we now want to verify whether other similar structural configurations can actually produce analogous results in term of electronic properties and consequent scanning probe results. As an example of this possibility, we here consider the case of a 5-atom $\mathrm{Cr}$ pyramid (four atoms in hollow sites above the surface layer and one above them) on $\operatorname{Cr}(100)$, which was simulated with the same numerical setup. In Fig. 5 we report the DOS of this tip model, which closely resembles the one previously shown for $\mathrm{Cr} / \mathrm{Cr}(100)$. Similarly to the single adatom case, it shows a pronounced peak at positive energies $(+0.3 \mathrm{eV})$ and a smaller feature at negative ones $(-0.6 \mathrm{eV})$. Consequently, the simulated $d I / d V$ curve is very close to the one of the $\mathrm{Cr} / \mathrm{Cr}(100)$ model, and to experiment. We conclude that both structural models are equally valid and represent good candidates for the real $\mathrm{Cr}$ apex in STS experiments.

\section{Spin sensitivity of bulk $\mathrm{Cr}$ tips}

An essential information in SP-STS experiments is the tip spin polarization, which can be obtained from $a b$ initio calculations, as shown in Fig. 2(b), but is not directly accessible to experiments. In the case of tip and sample with collinear magnetization axis, the most directly related measurable quantity is the so-called $d I / d V$ asymmetry, ${ }^{25}$ defined as follows:

$$
A(V)=\frac{d I / d V_{P}-d I / d V_{A P}}{d I / d V_{P}+d I / d V_{A P}},
$$

where $d I / d V_{P(A P)}$ is the differential conductivity acquired with the tip and sample magnetization axis pointing in parallel (antiparallel) direction. We note that also definitions leading to the opposite sign of $A$ are used. ${ }^{9,46}$ In the framework of the WKB model already used to interpret our spin-integrated results, it is possible to show that at zero bias $A$ is the product of the sample and the tip spin polarizations at the Fermi level, $A(0)=P_{s}(0) P_{t}(0),{ }^{25}$ allowing for the determination of $P_{t}(0)$ when the other quantities are known. At finite bias, the relation between $A, P_{s}$, and $P_{t}$ is in general not available, but one can approximate it as $A(V) \approx P_{s}(e V) P_{t}(0)$ or $A(V) \approx$ $P_{t}(-e V) P_{s}(0)$ by neglecting the tip or sample dependence on energy of the DOS and spin polarization. ${ }^{25,41,42}$ In analogy to these expressions, changes in $A(V)$ of a given sample as measured with various tips will be qualitatively described here in terms of changes in $P_{t}(-e V)$. As the most relevant 
advantage, this approach allows one to describe the spin polarization of the tunneling current using a voltage-dependent transmission coefficient and the sample and tip DOS evaluated at their respective surfaces. ${ }^{42}$ Despite its simplicity, this procedure was successfully used in the interpretation of SP-STS experiments. ${ }^{41-44}$ A more quantitative but computationally demanding approach would require the evaluation of tip and sample decaying of the wave functions and spin polarizations in the vacuum region ${ }^{10}$ and is beyond the aim of this work. In the following, we analyze experimental SP-STS data from the literature in order to extract information about the tip spin polarization. In particular, by considering some experimental $A(V)$ curves from the literature we aim to obtain an estimate of $P_{t}(0)$ and a qualitative behavior of $P_{t}(\mathrm{eV})$ to be compared with the calculations presented in Sec. III A. Although this approach is here applied to a specific system, the method is quite general and can be used to interpret a large variety of experiments.

Magnetic properties of bulk $\mathrm{Cr}$ tips were investigated in two different works. ${ }^{8,9}$ Schlenhoff et al. ${ }^{8}$ presented SP-STS measures on a 1.5 ML Fe film on W(110). The interpretation of their experimental data is however complicated by a bias-dependent tip-sample distance, ${ }^{43,45}$ following the $d I / d V$ acquisition, obtained in a constant current mode. Hence we focus on the SP-STS results by Corbetta et al.,${ }^{9}$ performed in a constant height mode on ferromagnetic Co islands on $\mathrm{Cu}(111)$. By applying an external magnetic field perpendicular to the surface plane, the authors obtained both an antiparallel and a parallel alignment between the sample and the tip magnetization axis. The experimental data of that work, which are of particular interest in our context, are reported in Fig. 6(a). Two measurements of $A(V)$ were obtained on the same Co island with two different apex structures from the same macroscopic $\mathrm{Cr}$ tip, referred to as tip $\alpha$ and tip $\beta$. One notices first that $\alpha$ and $\beta$ measurements have often opposite sign, especially in a region (highlighted in gray) where they are almost specular. Consequently, opposite tip polarizations can be expected in wide energy ranges. In particular, at zero bias, they obtained $P_{t}(0)=+0.45$ for tip $\alpha$ and $P_{t}(0)=-0.35$ for tip $\beta$. Next, both experimental $A(V)$ show the presence of sign changes (zeros) which where ascribed to the energy dependence of the sample spin polarization. ${ }^{9,46}$ Conversely, the differences in the position of zeros (which can be seen here around $V=-0.1$ and $+0.45 \mathrm{~V}$ ) can be attributed to different energy behaviors for $P_{t}(E)$ in the corresponding (reversed) energy windows, namely at about $E=+0.1$ and $-0.45 \mathrm{eV}$

The study of model tips presented in this work also shows how the sign of the spin polarization can change significantly depending on the structure of the apex. Tentatively, $\mathrm{Cr} / \mathrm{Cr}(111)$ with $P_{t}(0)=+0.61$ could be taken as a model to tip $\alpha$, and $\mathrm{Cr} / \mathrm{Cr}(100)$ with $P_{t}(0)=-0.60$ as a model to tip $\beta . \mathrm{Cr} / \mathrm{Cr}(110)$ is also qualitatively compatible with tip $\alpha$, although with a smaller value of $P_{t}(0)=0.21$, and will not be considered further. The energy dependence of $P_{t}$, previously shown in Fig. 2(b), is enlarged in Fig. 6(b) for better comparison. Both $P_{t}$ curves are smooth and without zeros in the shaded region. We recall that in this case the approximation $A(V) \approx P_{s}(e V) P_{t}(0)$ holds and specular $A(V)$ curves are expected, as those actually measured. Outside this
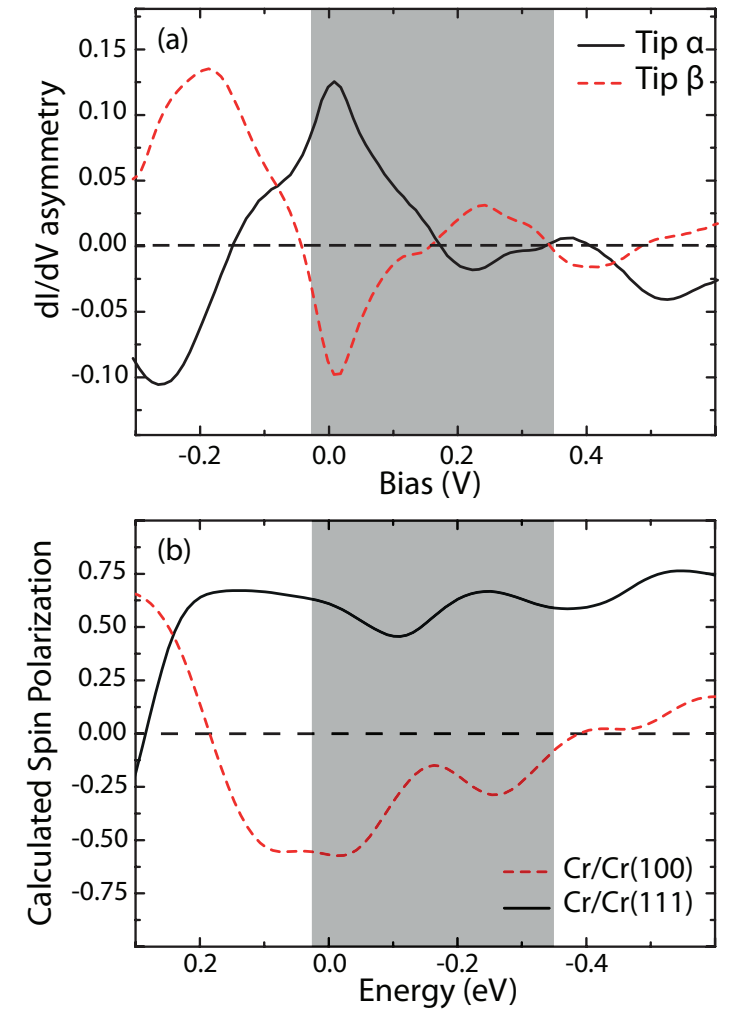

FIG. 6. (Color online) (a) $d I / d V$ asymmetry for Co islands on $\mathrm{Cu}(111)$ measured with two different $\mathrm{Cr}$ tips, as described in Ref. 9. Courtesy of Max Planck Institute of Microstructure Physics, Halle, Germany. We multiplied the original asymmetry curves by -1 to account for the different definition of asymmetry in Eq. (6) of our work as compared to Ref. 9. (b) Calculated $P_{t}(\mathrm{eV})$ for $\mathrm{Cr}$ atom on $\mathrm{Cr}(100)$ and $\mathrm{Cr}(111)$ structures, from Fig. 2(b). To compare experiments and theory making use of Eqs. (6) and (1), the energy scale is reversed, as described in the text.

range, calculated $P_{t}$ behave differently for the two models, which can be connected to the shift in the position of zeros discussed above. We can infer that the association of tip $\alpha$ with a $\mathrm{Cr} / \mathrm{Cr}(111)$ structure and of tip $\beta$ with $\mathrm{Cr} / \mathrm{Cr}(100)$ is consistent with all the main features experimentally reported.

We conclude this section by commenting on another SPSTM result from the recent literature. In our previous work, ${ }^{7}$ a first evidence of $\mathrm{Cr}$ tip in-plane spin sensitivity was obtained on a $\operatorname{Cr}(100)$ sample surface. Spin contrast in $d I / d V$ maps of adjacent $\mathrm{Cr}$ terraces was detected at a bias of $-0.3 \mathrm{~V}$. Although little information can be extracted from this single measurement, a tip with nonnegligible spin asymmetry at $E=$ $+0.3 \mathrm{eV}$ can account for such measurements. This is consistent with the calculated $P_{t}$ of both $\mathrm{Cr} / \mathrm{Cr}(100)$ and $\mathrm{Cr} / \mathrm{Cr}(110)$, but not of $\mathrm{Cr} / \mathrm{Cr}(111)$.

\section{CONCLUSIONS}

Combining DFT calculations and STS measurements, we characterized the electronic and magnetic properties of bulk Cr tips. A model of tunneling based on the 1D-WKB approximation was used to interpret experimental findings. Tip features at $+0.3 \mathrm{eV}$ and $-0.5 \mathrm{eV}$ were found in $d I / d V$ 
spectra from $\mathrm{Si}(111)-7 \times 7$ and $\mathrm{Au}(111)$. Among the calculated tip structures, $\mathrm{Cr} / \mathrm{Cr}(100)$ best accounts for the measured tip properties. Spin-polarized DFT calculations were compared to SP-STS experiments from the literature, evincing the role of the tip structure on the sign and the energy dependence of the spin polarization. Large values of tip polarization from our simulations are in agreement with experimental data from the literature and support the use of bulk Cr tips as efficient SP-STM and SP-STS probes. Our results confirm the importance of interpreting STS spectra by an appropriate model of tunneling, in particular when the dependence on energy of the tip DOS cannot be neglected. Moreover, the presented methods are relatively simple and general, which makes them interesting for interpreting a large variety of STS and SP-STS experiments.

\section{ACKNOWLEDGMENTS}

The authors are grateful to M. Corbetta, S. Ouazi, J. Borme, Y. Nahas, H. Oka, S. Wedekind, D. Sander, and J. Kirschner for providing the SP-STS data of the bulk Cr tip and thank C. E. Bottani, A. Picone, M. Finazzi, L. Duò, and F. Ciccacci for valuable discussions. This work was financed by Fondazione Cariplo through the project IMMAGINA (Grant No. Ref. 20085.2412) and Prot. 0018524, and by MIUR through PRIN Project No. 2007CMLFY2.
${ }^{1}$ A. J. Melmed, J. Vac. Sci. Technol. B 9, 601 (1991).

${ }^{2}$ M. Bode, M. Getzlaff, and R. Wiesendanger, Phys. Rev. Lett. 81, 4256 (1998).

${ }^{3}$ O. Pietzsch, A. Kubetzka, M. Bode, and R. Wiesendanger, Phys. Rev. Lett. 84, 5212 (2000).

${ }^{4}$ A. Kubetzka, M. Bode, O. Pietzsch, and R. Wiesendanger, Phys. Rev. Lett. 88, 057201 (2002).

${ }^{5}$ S. F. Ceballos, G. Mariotto, S. Murphy, and I. V. Shvets, Surf. Sci. 523, 131 (2003).

${ }^{6}$ R. Wiesendanger, H. J. Güntherodt, G. Güntherodt, R. J. Gambino, and R. Ruf, Phys. Rev. Lett. 65, 247 (1990).

${ }^{7}$ A. Li Bassi, C. S. Casari, D. Cattaneo, F. Donati, S. Foglio, M. Passoni, C. E. Bottani, P. Biagioni, A. Brambilla, M. Finazzi et al., Appl. Phys. Lett. 91, 173120 (2007).

${ }^{8}$ A. Schlenhoff, S. Krause, G. Herzog, and R. Wiesendanger, Appl. Phys. Lett. 97, 083104 (2010).

${ }^{9}$ M. Corbetta, S. Ouazi, J. Borme, Y. Nahas, F. Donati, H. Oka, S. Wedekind, D. Sander, and J. Kirschner, Jpn. J. Appl. Phys. 51, 030208 (2012).

${ }^{10}$ P. Ferriani, C. Lazo, and S. Heinze, Phys. Rev. B 82, 054411 (2010).

${ }^{11}$ M. Passoni, F. Donati, A. Li Bassi, C. S. Casari, and C. E. Bottani, Phys. Rev. B 79, 045404 (2009).

${ }^{12}$ B. Koslowski, H. Pfeifer, and P. Ziemann, Phys. Rev. B 80, 165419 (2009).

${ }^{13}$ J. P. Perdew, K. Burke, and M. Ernzerhof, Phys. Rev. Lett. 77, 3865 (1996).

${ }^{14}$ S. A. Werner, A. Arrott, and H. Kendrick, Phys. Rev. 155, 528 (1967).

${ }^{15}$ P. E. Blöchl, Phys. Rev. B 50, 17953 (1994).

${ }^{16}$ G. Kresse and D. Joubert, Phys. Rev. B 59, 1758 (1999).

${ }^{17}$ G. Kresse and J. Furthmüller, Phys. Rev. B 54, 11169 (1996).

${ }^{18}$ D. J. Singh and J. Ashkenazi, Phys. Rev. B 46, 11570 (1992).

${ }^{19}$ J. A. Stroscio, D. T. Pierce, A. Davies, R. J. Celotta, and M. Weinert, Phys. Rev. Lett. 75, 2960 (1995).

${ }^{20}$ G. Bihlmayer, T. Asada, and S. Blügel, Phys. Rev. B 62, R11937 (2000).

${ }^{21}$ J. V. Barth, H. Brune, G. Ertl, and R. J. Behm, Phys. Rev. B 42, 9307 (1990).

${ }^{22}$ W. A. Hofer, J. Redinger, A. Biedermann, and P. Varga, Surf. Sci. 482-485, 1113 (2001).

${ }^{23}$ W. A. Hofer and A. J. Fisher, Surf. Sci. 498, L65 (2003).
${ }^{24}$ H. Zabel, J. Phys.: Condens. Matter 11, 9303 (1999).

${ }^{25}$ D. Wortmann, S. Heinze, P. Kurz, G. Bihlmayer, and S. Blügel, Phys. Rev. Lett. 86, 4132 (2001).

${ }^{26}$ V. A. Ukraintsev, Phys. Rev. B 53, 11176 (1996).

${ }^{27}$ B. Koslowski, C. Dietrich, A. Tschetschetkin, and P. Ziemann, Phys. Rev. B 75, 035421 (2007).

${ }^{28}$ G. Hörmandinger, Phys. Rev. B 49, 13897 (1994).

${ }^{29}$ F. Donati, S. Piccoli, C. E. Bottani, and M. Passoni, New J. Phys. 13 (2011).

${ }^{30}$ M. Passoni and C. E. Bottani, Phys. Rev. B 76, 115404 (2007).

${ }^{31}$ G. Binnig, H. Rohrer, C. Gerber, and E. Weibel, Phys. Rev. Lett. 50, 120 (1983).

${ }^{32}$ R. Wolkow and P. Avouris, Phys. Rev. Lett. 60, 1049 (1988).

${ }^{33}$ K. Takayanagi, Y. Tanishiro, M. Takahashi, and S. Takahashi, J. Vac. Sci. Technol. A 3, 1502 (1985).

${ }^{34}$ Y. L. Wang, H. J. Gao, H. M. Guo, H. W. Liu, I. G. Batyrev, W. E. McMahon, and S. B. Zhang, Phys. Rev. B 70, 073312 (2004).

${ }^{35}$ R. J. Hamers, R. M. Tromp, and J. E. Demuth, Phys. Rev. Lett. 56, 1972 (1986).

${ }^{36}$ R. J. Hamers, R. M. Tromp, and J. E. Demuth, Surf. Sci. 181, 346 (1987).

${ }^{37}$ P. Avouris and R. Wolkow, Phys. Rev. B 39, 5091 (1989).

${ }^{38}$ P. Sutter, P. Zahl, E. Sutter, and J. E. Bernard, Phys. Rev. Lett. 90, 166101 (2003).

${ }^{39}$ L. C. Davis, M. P. Everson, R. C. Jaklevic, and W. Shen, Phys. Rev. B 43, 3821 (1991).

${ }^{40}$ J. Kliewer, R. Berndt, E. V. Chulkov, V. M. Silkin, P. M. Echenique, and S. Crampin, Science 288, 1399 (2000).

${ }^{41}$ T. K. Yamada, M. M. J. Bischoff, G. M. M. Heijnen, T. Mizoguchi, and H. van Kempen, Phys. Rev. Lett. 90, 056803 (2003).

${ }^{42}$ K. Palotás, W. A. Hofer, and L. Szunyogh, Phys. Rev. B 83, 214410 (2011).

${ }^{43}$ K. Palotás, W. A. Hofer, and L. Szunyogh, Phys. Rev. B 84, 174428 (2011).

${ }^{44}$ K. Palotás, W. A. Hofer, and L. Szunyogh, Phys. Rev. B 85, 205427 (2012).

${ }^{45}$ M. Ziegler, N. Néel, A. Sperl, J. Kröger, and R. Berndt, Phys. Rev. B 80, 125402 (2009).

${ }^{46}$ H. Oka, P. A. Ignatiev, S. Wedekind, G. Rodary, L. Niebergall, V. S. Stepanyuk, D. Sander, and J. Kirschner, Science 327, 843 (2010). 